\title{
Radial and Nonradial Effects of Radial Fields in Frenet Frame
}

\author{
Jakub Czajko \\ Science/Mathematics Education Department \\ Southern University and A\&M College \\ P.O. Box 64998, Baton Rouge, LA 70896, USA \\ Tel: 936-648-4447_E-mail: sunswing77@netscape.net
}

Received: February 22, 2011 Accepted: March 11, 2011 doi:10.5539/apr.v3n1p2

\begin{abstract}
Frenet-Serret formulas applied to the concept of work done or to the corresponding to it notion of potential energy in radial/center-bound force field imply coexistence of radial effects with certain nonradial effects of the radial field, regardless of whether the field is of gravitational nature or not.
\end{abstract}

Keywords: Potential energy, Work done, Force field, Nonradial effects of gravity

\section{Introduction}

Rate of change of work done $\mathrm{dW} / \mathrm{dt}$ is usually defined as change of energy $\mathrm{E}$ in time $\mathrm{t}$ (i.e. power), which equals to scalar/dot product of force and velocity vectors. Hence the change of work done dW equals to the dot product of the force field vector $\mathbf{F}$ and the differential $\mathrm{d} \mathbf{r}$ (of a test particle's trajectory radius vector $\mathbf{r}$ ) pointing in the direction of the force $\mathbf{F}$ that acts on the given particle:

$$
\mathrm{dW}=\mathrm{dE}=\mathbf{F} \cdot \mathrm{d} \mathbf{r} \quad \Leftarrow \quad \mathrm{dW} / \mathrm{dt}=\mathrm{dE} / \mathrm{dt}=\mathbf{F} \cdot \mathbf{v}
$$

where $\mathbf{v}=\mathrm{d} \mathbf{r} / \mathrm{dt}$ denotes instantaneous velocity vector tangent to the trajectory - compare Parrott (1987). If the velocity vector would act at an angle $\alpha$ with respect to the direction of the force field $\mathbf{F}$, then its effective value would diminish as $\mathrm{v} \cos \alpha$, where the scalar $\mathrm{v}=\|\mathbf{v}\|=\|\mathrm{dr} / \mathrm{dt}\|=\mathrm{dr} / \mathrm{dt}$ signifies the speed that corresponds to the velocity vector $\mathbf{v}$. The scalar/dot product of vectors is denoted by bold dot, whereas regular multiplication by numbers/functionals/functions/operators is implied. The arrow signs: $\Leftrightarrow, \Leftarrow, \Rightarrow$ mark, respectively: formal equivalence and (oriented) logical implications of forms of statements/expressions/formulas.

Although the conventional expression for speed $\mathrm{v}=\mathrm{dr} / \mathrm{dt}$ is commonly used in physics and applied mathematics, Shilov (1996) pointed out that the length of the vector specifying the tangent has no direct geometrical meaning. In astrophysics, however, where exact trajectories of particles are not always exactly known in advance, they cannot always be determined theoretically with precision necessary for reconciliation of experimental data. Yet the point of origin of photons arriving from distant stars can be pinpointed with sufficient precision and thus the use of a pointing vector for determination of their projected trajectory seems quite natural. In the sense also the length of the radius vector specifying their trajectory is physically meaningful, because it can be interpreted as the distance to the particle at any given point on the trajectory. One could think of the pointing vector as determining the radial distance from the local center of gravity to the test particle that traverses the body's gravitational field. This interpretation is inportant for observations of rays coming from distant stars when their paths were distorted by some "third" massive body moving nearby the rays' trajectory path. Therefore, a certain "new synthetic" approach to determination of the external distortion of the rays' trajectory in $\mathrm{radial} / \mathrm{center-bound} \mathrm{force} \mathrm{fields} \mathrm{is} \mathrm{proposed} \mathrm{below.}$

Calculating gravitational frequency shift is feasible when only difference between two potentials: on the star emitting the rays and on the Earth is being considered - see Beiser (1973). But when rays from Taurus A passed by our Sun near occultation, they experienced an extra, unanticipated frequency decrease caused by our Sun's field - see Sadeh, Knowles \& Yaplee (1968). However, the essentially radial, gravitational frequency shift that Einstein proposed proved quite irrelevant in this case, because the blueshift that the rays acquired on their incoming trajectory (towards our Sun) before their perihelion was practically cancelled out by the redshift the rays regained on their outgoing trajectory (with respect to our Sun) after passing their perihelion, given the fact that the extraneous frequency decrease appeared only up to a few solar radii from the surface of our Sun. 
Sadeh, Knowles \& Au (1968) also have reported quite similar unanticipated yet clearly distance-dependent frequency decrease in radio waves triggered by a set of atomic clocks resting on the Earth's surface once the waves were intercepted by another set of atomic clocks riding a truck at relativistically insignificant speed on a highway.

Both the extraneous frequency decrease effects appeared along equipotential surfaces of our Sun and Earth, respectively, where gravitational potentials remain constant for most practical purposes. Szekeres (1968) pointed out that those experiments could not be explained by any gravitational or electromagnetic theory of physics. Eventually Czajko (2000) has retrodicted the results of those two experiments through a new theory conjecturing existence of certain nonradial effects of the usual radial/center-bound gravitational force field. Recall that Einstein (1916) specifically omitted any deviations [from radial gravity] due to some [possible, at least theoretically] tangential effects, because they would be too slight when measured on the Earth's surface.

The relativistic deflection of light that Einstein discovered is also not too helpful for determination of trajectory of the rays coming from Taurus A, because several unbiased and quite independent observations (see Merat, 1974) detected rather significant excess over the Einstein's prediction of deflection of light estimated at 10-15\%. As a matter of fact, the excess has also been retrodicted by the new theory of nonradial effects of gravity proposed by Czajko (2000).

Therefore, perhaps a quite new, strictly mathematical approach to exact determination of generally unknown trajectory path in purely differential-geometric terms within radial force fields (whether of gravitational or electromagnetic nature) is needed. Precision of such a theoretically exact new formula will depend on data from measurements as well as on estimates of physical parameters of all the sources of the local force fields involved, of course, but its generic mathematical exactness in absence of detailed knowledge of the trajectory's shape is what should make the new formula applicable to situations in which extraneous or formerly quite unexpected/unpredicted physical phenomena happen.

Such a generic approach to abstract curves treated as locally distorted by an externak influence regardless of detailed knowledge of their associated equations has already been started in classical differential geometry by Frenet (1852) and also Serret (1851). They used fairly simple geometric reasonings to prove the point that any curve in three-dimensional (3D) space can be represented by a trihedron moving along the very curve, whose twisting can be determined by just three mutually orthogonal generic unit-length vectors representing fixed arms of the trihedron.

Therefore, without detailed knowledge of the equation representing particular trajectory curve, all active variables describing physical and geometric objects should be expressed as certain generic functions, whose exact formal representation could then be determined directly by some relevant physical constraints later rather than by purely geometric terms of abstract vector spaces.

Hence if the speed $\mathrm{v}$ should represent a certain scalar speed function $\mathrm{v}()$, then the expression $\mathrm{dr} / \mathrm{dt}$ should actually be rendered as derivative $\operatorname{dr}() / d t$ of some generic function $\mathrm{r}()$ of the pointing vector function $\mathbf{r}()$ also known as the radius function of the trajectory, which is supposed to be actually differentiated. Henceforth any function $\mathrm{f}$ will be denoted by $\mathrm{f}()$ in order to distinguish it from a simple variable by the same name $\mathrm{f}$ or from a functional $\mathrm{f}$ of the function. Note that functional relationship between variables in a function is independent of choice of a coordinate system. Only representations of functions may differ, depending on coordinates.

Yet another important reason for distinguishing functions from their functionals, which are just values of their functions to be calculated from certain initial conditions, is that vectors and their first differentials are mutually orthogonal, which fact is well known and respected in mathematics, but it is oftentimes disregarded in physics, even if quite inadvertently. The use of functionals and scalar functions alone not always makes that fact clear.

Most general and generic equation of motion along an unspecified curve within three-dimensional Euclidean space can be expressed via frame moving along generally double-curved trajectory path with the use of abstract Frenet-Serret formulas, which determine the moving trihedron/tripod, and take the following form for unit speed - compare: Struik (1988):

$$
\begin{aligned}
& \mathrm{d} \mathbf{T}()=\kappa \mathbf{N} \mathrm{ds} \\
& \mathrm{d} \mathbf{N}()=-\kappa \mathbf{T} \mathrm{ds}+\tau \mathbf{B} \mathrm{ds} \\
& \mathrm{d} \mathbf{B}()=-\tau \mathbf{N} \mathrm{ds}
\end{aligned}
$$


when expressed by functions of the unit-length vectors $\mathbf{T}, \mathbf{N}, \mathbf{B}$ which are the tangent, principal normal and binormal vectors, respectively, of the Frenet frame $(\mathbf{T}, \mathbf{N}, \mathbf{B})$ also known as trihedron whose stance can lean as it moves along the $3 \mathrm{D}$ trajectory curve. Note that the twin scalar products $\mathbf{T} \cdot \mathbf{T}=\mathbf{N} \cdot \mathbf{N}=\mathbf{B} \cdot \mathbf{B}=1$, and $\mathbf{T} \cdot \mathbf{N}=\mathbf{T}$ - $\mathbf{B}=\mathbf{N} \cdot \mathbf{B}=0$, regardless of order. The unit-length vectors as well as primary curvature $\kappa$ and torsion $\tau$ (i.e. the second curvature that always stands perpendicular to the primary one), can be represented also in differential terms of abstract generic motion as certain kinematic functions of linear and/or angular speeds and accelerations. Here $\mathrm{s}$ is the curvilinear distance measured along the unspecified trajectory path that is being determined on the fly via moving trihedron whose twisting is due to certain external influences symbolized in the Frenet-Serret formulas by the nonspecific curvature and torsion.

Differentials of functions of single variable may appear without parentheses on the right-hand side (RHS) of equations. Hence equating $\mathrm{dr}()=\mathrm{dr}$ is fine for a scalar function $\mathrm{r}(\mathrm{)}$, but differentials of multi-variable functions, such as vectors, require product differentiation rule to be applied first.

\section{Geometrical expression of work done viewed from a moving trihedron}

Instead of expressing the trajectory's radius vector function $\mathbf{r}()$ in terms of an unspecified internal reference frame, it should be represented within the trihedron that is external to the source of the given force field. Inside the reference frame that is typically associated with the local center of the gravitational force field, direct actions exerted by the field are obviously radial, but the trajectory of a test particle that neither formally nor practically contributes to the dominant field (because the particle is usually too small as compared to the source that dominates the force field) its formal presence is virtually "invisible" therein. Therefore the impact of the radial force field should be expressed within the trihedron that moves with the particle along its trajectory. If one would still prefer to put it in the center-bound frame, it should be dereferenced from the trihedron anyway.

Since vectors are objects with three independent attributes/variables: arrow, direction and length, all of the attributes should be directly represented in a most generic vector function. Hence the radius vector function $\mathbf{r}()$ of a test particle's trajectory can be standardized inside the trihedron as:

$$
\mathbf{r}()=\varepsilon \mathbf{N}() \mathrm{r}()
$$

where $\mathrm{r}()=\|\mathbf{r}()\|$ is a length function of the trajectory radius (or pointing) vector and the functional $\varepsilon:=\cos (\mathbf{r}, \mathbf{N})$ denotes the vector's arrow represented here by planar angle between the radius and the principal normal vector. The compound sign:= means substitution of value of a functional (or number), which does not participate in the differentiation, because $\mathrm{d}(\mathrm{nf}())=\mathrm{ndf}()$ for any number $\mathrm{n}$ and any function $\mathrm{f}()$. The substitution sign is used in some programming languages in order to distinguish equating from substituting, which are distinct and different operations, and mixing of which might lead to serious conceptual errors or even misconceptions. Some dangers of making mistakes during abstract conceptualizations in pure mathematics were exposed by Šanin (1968).

Differentiation of the radius vector function $\mathbf{r}()$ defined above yields the differential function $\mathrm{d} \mathbf{r}()$ :

$$
\mathrm{d} \mathbf{r}()=\varepsilon[\mathbf{N d r}()+\operatorname{rdN}()]=\varepsilon[\mathbf{N d r}-\mathbf{T r} \kappa d s+\mathbf{B r} \tau \mathrm{ds}]=\varepsilon[\mathbf{N d r}-\operatorname{Trd} \varphi+\mathbf{B r d} \Theta]
$$

where $\kappa=\mathrm{d} \varphi / \mathrm{ds}$ is the primary curvature, $\varphi$ is the tangential angle set in the equatorial plane of the (presumably rotating) field's source, $\tau=\mathrm{d} \Theta / \mathrm{ds}$ is the torsion, and $\Theta$ is the binormal angle set in one of the source's polar planes - compare Larson \& Hostetler (1986). As outcome of differentiation, the unit-length vector functions can be represented by their functionals T, N, B, on the RHS of the equation (2.2). Although terms like 'equatorial' and 'polar' are not used in differential geometry in reference to planes or trihedron, they seem very well suited to be applied to situations encountered in astrophysics or physics, because of their conceptual proximity to the terms used for describing the rotating masses that generate gravitational force fields, and extend to the trihedron.

Now in order to match the trihedron's unit-length vectors, the force field vector must also be cast onto the trihedron. Apart from rotation of the field's source, the impact of which shall be shown elsewhere, the most general (spatial/directional) distribution (or directional decomposition) of the radial/center-bound force field vector function $\mathbf{F}($ ) can be represented within the trihedron as:

$$
\mathbf{F}()=\mathbf{F}_{\mathrm{N}}()+\mathbf{F}_{\mathrm{T}}()+\mathbf{F}_{\mathrm{B}}()=\varepsilon_{\mathrm{N}}() \mathbf{N F}_{\mathrm{N}}()+\varepsilon_{\mathrm{T}}() \mathbf{T F}_{\mathrm{T}}()+\varepsilon_{\mathrm{B}}() \mathbf{B} \mathrm{F}_{\mathrm{B}}()
$$

where each alignment coefficient function $\varepsilon_{\mathrm{N}}()=\cos (\mathbf{N}, \mathbf{F}), \varepsilon_{\mathrm{T}}()=\cos (\mathbf{T}, \mathbf{F}), \varepsilon_{\mathrm{B}}()=\cos (\mathbf{B}, \mathbf{F})$ shall reflect the impact of the angle between each of the trihedron's arms and the respective directional force subcomponent $\mathbf{F}_{\mathrm{i}}()$ where $\mathrm{i}:=\mathrm{N}, \mathrm{T}, \mathrm{B}$. The scalars $\mathrm{F}_{\mathrm{i}}()$ denote thus the values of the respective directional subcomponents of the force field vector cast onto the moving trihedron. 
Replacing the equation (1.1) by the dot product of the force field vector (2.3) and the differential of the radius vector function (2.2) yields change of work done as seen from the trihedron:

$$
\mathrm{dW}=\varepsilon\left[\varepsilon_{\mathrm{N}}() \mathrm{F}_{\mathrm{N}}() \mathrm{dr}-\varepsilon_{\mathrm{T}}() \mathrm{F}_{\mathrm{T}}() \mathrm{rd} \varphi+\varepsilon_{\mathrm{B}}() \mathrm{F}_{\mathrm{B}}() \mathrm{rd} \Theta\right] \quad \Leftrightarrow \quad \mathrm{dW}_{\mathrm{N}}()+\mathrm{dW}_{\mathrm{T}}()+\mathrm{dW}_{\mathrm{B}}()
$$

where the arrow $\varepsilon:=\cos (\mathbf{r}, \mathbf{N})$ just as before. In general, thus, change of the work done function, as seen from a moving trihedron, can contain not only the usual radial=normal component $\mathrm{dW}_{\mathrm{N}}$, but also certain nonradial (i.e. tangential $\mathrm{dW}_{\mathrm{T}}$ and binormal $\mathrm{dW}_{\mathrm{B}}$ ) components even though the force field vector is always radial within the radial/center-bound reference frame of a nonrotating local force field generated by a single (spheroidal) source mass/charge. Since only purely mathematical premises have been used above, the generic equation (2.4) applies to any radial/center-bound force field, not just to gravitational one. Although the equation (2.4) is valid only inside the moving trihedron, which can be perceived as dual to the center-bound reference frame within local force field, quite analogous equation for the center-bound reference frame shall be derived elsewhere.

\section{Dynamical expression of work done viewed from a moving trihedron}

Let us consider photon of a (negligibly small) mass $\mathrm{m}$ coming from a distant star along a trajectory path that is assumed as being practically straightlinear at first, but then it is deflected by our Sun (of mass M) which the photon passes by near occultation. Therefore the gravitational field of our Sun shifts the photon's frequency before the photon is intercepted on Earth. The extra nonradial impact of our Sun's presence near the photon's trajectory comes in addition to the gravitational redshift caused by difference between gravitational potential of the photon's distant home star and the Earth, which is the [radial] gravitational redshift that Einstein predicted.

At the photon's perihelion the radial subcomponent of the gravitational force field exerted by our Sun amounts to the entire force function: $\mathrm{F}_{\mathrm{N}}(\mathrm{r})=-\mathrm{GMm} / \mathrm{r}^{2}$ of the Sun's field, because only the radial=normal component of the Sun's force field acts thereat. The minus sign indicates that the (attractive) force is directed towards our Sun, hence away from the traversing photon. Although the gravitational force field is anchored at our Sun's gravity center, it is not pointing outward but inwards and that is the reason for reversing its sign/arrow in situations where directionality counts - compare Clausius (1885). Hence the total amount of the radial=normal part of the work done by our Sun's force field at the perihelion that corresponds to the radial field's component is given by:

$$
\mathrm{W}_{\mathrm{N}}:=\varepsilon \varepsilon_{\mathrm{N}} \int \mathrm{F}_{\mathrm{N}}(\mathrm{r}) \mathrm{dr}=-\int\left(-\mathrm{GMm} / \mathrm{r}^{2}\right) \mathrm{dr}=-\mathrm{GMm} / \mathrm{r}
$$

which is exactly the same as the traditional [radial] gravitational potential energy - see Halliday \& Resnick (1974). Here $r$ means the (radial) distance from our Sun's center of gravity to the photon's perihelion and G is the gravitational constant functional. At perihelion the radius and the principal normal vectors are parallel, but they point in opposite directions: $\varepsilon:=\cos (\mathbf{r}, \mathbf{N})=\cos \left(180^{\circ}\right)=-1$. The principal normal unit-length vector and the radial=normal component of the force vector also are parallel, but at the perihelion they point in the same direction (towards our Sun) so that one gets $\varepsilon_{\mathrm{N}}:=\cos \left(\mathbf{N}, \mathbf{F}_{\mathrm{N}}\right)=\cos \left(0^{\circ}\right)=1$. As seen within the trihedron at the photon's perihelion, the work done by our Sun's gravitational field against the photon (which is far too small to make any practically perceivable contribution to the local gravitational force field that is dominated by our Sun) equals to the whole amount of the Sun's gravitational potential energy at that point.

When the photon is still far away from our Sun (at a practically almost "infinitely" distant point where the tangential force field subcomponent in a practical limit equals to the entire force field of our Sun: $\mathrm{F}_{\mathrm{T}}:=-\mathrm{GMm} / \mathrm{r}^{2}$ ) the tangential part $\mathrm{W}_{\mathrm{T}}$ of the total amount of work done by our Sun's gravitational field against the photon in our Sun's equatorial plane as seen within the trihedron is:

$$
\mathrm{W}_{\mathrm{T}}:=-\operatorname{sign}(\varepsilon) \mathrm{K}_{\mathrm{T}} \mathrm{r} \mathrm{F}_{\mathrm{T}} \int \mathrm{d} \varphi=\mathrm{K}_{\mathrm{T}}\left(-\mathrm{rGMm} / \mathrm{r}^{2}\right) \int \mathrm{d} \varphi=-\mathrm{K}_{\mathrm{T}} \mathrm{GMm} \varphi / \mathrm{r}
$$

where the integration over the tangential angle $\varphi$ runs from zero to $\varphi^{\circ}[\mathrm{deg}]$ in our Sun's equatorial plane. In this case both the tangential force component $\mathrm{F}_{\mathrm{T}}$ and the effective overall local tangential alignment coefficient $\mathrm{K}_{\mathrm{T}}:=$ $\|\varepsilon\| \varepsilon_{\mathrm{T}} /[\mathrm{deg}]$ are devoid of the tangential angle $\varphi$ that signifies here the angular tangential=equatorial distance. Far away from our Sun the radial gravitational force of the Sun's field gets (practically) almost perfectly aligned along the tangent of the photon's trajectory, path, but its overall impact on the distant photon is diminishing with increasing regular (i.e. radial as opposed to angular) distance to our Sun. There $\operatorname{sign}(\varepsilon)=-1$ because the angle $(\mathbf{r}, \mathbf{N})$ falls in the range of $\left(90^{\circ}, 180^{\circ}\right]$, and the tangential alignment coefficient of the field equals to $\varepsilon_{\mathrm{T}}:=$ $\cos \left(\mathbf{T}, \mathbf{F}_{\mathrm{T}}\right)$.

The standalone (hence: $F_{B}:=-G M m / r^{2}$ ) binormal constituent $W_{B}$ (which is very important for fast rotating field's source) of the total amount of work done by the radial gravitational force field of our Sun against a distant photon (when the photon is still far away from its perihelion) equals to: 


$$
\mathrm{W}_{\mathbf{B}}:=\mathrm{K}_{\mathrm{B}} \mathrm{r} \mathrm{F}_{\mathrm{B}} \int \mathrm{d} \Theta=\mathrm{K}_{\mathrm{B}}\left(-\mathrm{rGMm} / \mathrm{r}^{2}\right) \int \mathrm{d} \Theta=-\mathrm{K}_{\mathrm{B}} \mathrm{GMm} \Theta / \mathrm{r}
$$

where the integration over the binormal angle $\Theta$ would run from zero to $\Theta^{\circ}[\mathrm{deg}]$ in a polar plane of our Sun. The effective overall local binormal alignment coefficient $K_{B}:=\varepsilon \varepsilon_{B} /[\mathrm{deg}]$, with the binormal alignment coefficient $\varepsilon_{\mathrm{B}}:=\cos \left(\mathbf{r}, \mathbf{F}_{\mathrm{B}}\right)$, and the binormal force field component $\mathrm{F}_{\mathrm{B}}$ being devoid of the binormal angle $\Theta$. Formwise, these two nonradial formulas for work done comply with the formerly quite unanticipated nonradial effect, namely the unexpected frequency decrease in photons coming from Taurus A (Sadeh, Knowles \& Yaplee, 1968 ) as well as in radio waves generated on the Earth (Sadeh, Knowles \& Au, 1968). The nonradial effects were caused by the gravitational fields of our Sun and the Earth, respectively, as demonstrated by Czajko (2000).

The extra frequency decrease found in those experiments has been reconciled to relatively high degree of accuracy $\left(\mathrm{K}_{\text {Sun }} / \mathrm{K}_{\text {Earth }} \approx 0.4 \%\right)$ - given such different circumstances when a generalized equation corresponding to the local equations (3.2) and (3.3) was augmented by mass densities of the respective celestial bodies (our Sun and Earth - see Czajko, 2000), the need for which was also found for heat flow by Kellog (1929). Moreover, the very same generalized nonradial formula retrodicted the (experimentally confirmed) excess over Einstein's prediction of deflection of light (Czajko, 2000). It also supplied mathematical reason for a certain intrinsic gravitational repulsion that could cause the apparently accelerated expansion of the universe that was inferred from new observational data - see Czajko (2004). Furthermore, presence of the nonradial effects of gravity could explain also certain other, previously unreconciled experiments with flying atomic clocks - see Czajko (1990), and Czajko (1991).

\section{Conclusions}

Work done in three-dimensional space comprising nonrotating radial/center-bound gravitational force field admits certain nonradial (tangential and binormal) effects in addition to the usual radial effects of the radial field. The nonradial effects occur only along equipotential surfaces (where the radial potential is always constant) in the equatorial and polar planes of a local force field's source mass/charge, respectively. The nonradial effects of the radial force field are thus mathematically necessary consequences of the very presence of the $\mathrm{radial} / \mathrm{center-bound}$ force field (of any kind - not only gravitational one) alone.

\section{References}

Beiser, A. (1973). Concepts of modern physics. New York: McGraw-Hill Book Co., p.67ff.

Clausius, R. (1885). Potentialfunction und das Potential. Ein Beitrag zur mathematischen Physik. Leipzig: Johann Ambrosius Barth, p.2ff.

Czajko, J. (1990). On the Hafele-Keating experiment. Ann. Phys. (Leipzig), 47, 517-518.

Czajko, J. (1991). Experiments with flying atomic clocks. Exper. Tech. Phys. 39, 145-147.

Czajko, J. (2000). On conjugate complex time II: Equipotential effect of gravity retrodicts differential and predicts apparent anomalous rotation of the Sun. Chaos, Solit. Fract, 11, 2001-2016.

Czajko, J. (2004). On the intrinsic gravitational repulsion. Chaos, Solit. Fract, 20, 683-700.

Einstein, A. (1916). The Foundations of the General Theory of Relativity [pp.111-164 in: H.A. Lorentz et al. (1923). The principle of relativity. New York: Dover, see p.161].

Frenet, F. (1852). Sur les courbes à double courbure. Journal des mathématiques pures et appliquées $1^{\mathrm{re}}$ série t.17 p.437-447 [Online] Available: http://portail.mathdoc.fr/JMPA/afficher_notice.php?id=JMPA_1852_1_17_ A22_0.

Halliday, D. \& Resnick, R. (1974). Fundamentals of physics. New York: J. Wiley \& Sons, p.266.

Kellog, O.D. (1929). Foundations of potential theory. Berlin: Verlag von Julius Springer, p.77.

Larson, R.E., \& Hostetler, R.P. (1986). Calculus with analytic geometry. Lexington, MA: D.C. Heath \& Co., p.761.

Merat, P. (1974). Observed Deflection of Light by the Sun as a Function of Solar Distance. Astron. Astrophys, 32 471-475.

Parrott, S. (1987). Relativistic electrodynamics and differential geometry. New York: Springer-Verlag, p.22f.

Sadeh, D., Knowles, S. \& Au, B. (1968). The effect of mass on frequency. Science, 161, 567-569.

Sadeh, D., Knowles, S.H. \& Yaplee, B.S. (1968). Search for a frequency shift of the 21-centimeter line from Taurus A near occultation by Sun. Science, 159, 307-308. 
Šanin, N.A. (1968). Constructive real numbers and constructive function spaces. Providence, RI: Am. Math. Society, pp.305f, 307.

Serret, J.-A. (1851). Sur quelques formules relatives à la théorie des courbes à double courbure. Journal des mathématiques pures et appliquées $1^{\mathrm{re}}$ série t.16 p.193-207 [Online] Available: http://portail.mathdoc.fr/JMPA/afficher_notice.php?id=JMPA_1851_1_16_A12_0.

Shilov, G.E. (1996). Elementary functional analysis. Mineola, NY: Dover, p.190.

Struik, D.J. (1988). Lectures on classical differential geometry. New York: Dover, p.18f.

Szekeres, G. (1968). Effect of gravitation on frequency. Nature, 220, 1116-1118. 